\title{
The management of paravalvular leaks post aortic valve replacement.
}

\author{
Shalini Kananathan ${ }^{1}$, Lakshini Perera ${ }^{1}$, Maneeshaa Mohanarajan ${ }^{1}$, Mohamed Sherif ${ }^{2}$, and \\ Amer Harky ${ }^{3}$ \\ ${ }^{1}$ Dnipropetrovsk Medical Institute of Conventional and Alternative Medicine \\ ${ }^{2}$ Northern General Hospital \\ ${ }^{3}$ Liverpool Heart and Chest Hospital NHS Foundation Trust
}

September 24, 2021

\begin{abstract}
Paravalvular leak (PVL) is uncommon but can lead to severe complications after surgical or transcatheter aortic valve replacement. Clinical complications such as heart failure, haemolysis and infective endocarditis can be catastrophic results if not treated in promptly. It is, therefore, vital that PVLs are diagnosed early using various imaging modalities. Different approaches have been studies in managing PVL's; of late, there is an increased interest in the use of minimally invasive procedures such as the transcatheter aortic valve closure procedure due to the decreased occurrence of further operations. This review discusses the classification of PVLs, diagnostic approaches and the available management options.
\end{abstract}

The management of paravalvular leaks post aortic valve replacement.

Shalini Kananathan BSc ${ }^{1}$, Lakshini Perera BSc${ }^{2}$, Maneeshaa Mohanarajan BSc ${ }^{3}$, Mohamed Sherif MBBCh, MRCS $^{4}$, Amer Harky MSc, MRCS ${ }^{5}$

1. Dnipro Medical Institute of Traditional and Non-Traditional Medicine, Dnipro, Ukraine

2. Dnipro Medical Institute of Traditional and Non-Traditional Medicine, Dnipro, Ukraine

3. Dnipro Medical Institute of Traditional and Non-Traditional Medicine, Dnipro, Ukraine

4. Department of Cardiothoracic Surgery, Northern General Hospital, Sheffield, UK

5. Department of Cardiothoracic Surgery, Liverpool Heart and Chest Hospital, Liverpool, UK

Corresponding authors:

Amer Harky

MSc, MRCS

Department of Cardiothoracic surgery

Liverpool Heart and Chest Hospital

Liverpool, UK E-mail: amer.harky@lhch.nhs.uk

Phone: +44-151-600-1616

Funding: none obtained

Conflict of interest: all authors declare no conflict of interest

Word Count: 3118 


\section{Abstract}

Paravalvular leak (PVL) is uncommon but can lead to severe complications after surgical or transcatheter aortic valve replacement. Clinical complications such as heart failure, haemolysis and infective endocarditis can be catastrophic results if not treated in promptly. It is, therefore, vital that PVLs are diagnosed early using various imaging modalities. Different approaches have been studies in managing PVL's; of late, there is an increased interest in the use of minimally invasive procedures such as the transcatheter aortic valve closure procedure due to the decreased occurrence of further operations. This review discusses the classification of PVLs, diagnostic approaches and the available management options.

\section{Introduction}

PVL is not a common but severe complication that develops post-surgical and transcatheter aortic value replacement. PVLs occur due to a gap being present between the native annulus and the prosthetic valve's outer edge ${ }^{1}$. When the prosthetic valve detaches, it forms a channel between the implanted valve and the cardiac tissue through which blood flows; the flow of blood through this channel is referred to as a $\mathrm{PVL}^{2}$. PVL is caused by sewing ring suture disruption that can be triggered by infective endocarditis which may be associated with abscess formation, annulus fibrotic scars or calcification. Although clinically silent in most cases, PVLs can also develop into severe regurgitation, which manifest into clinical symptoms of heart failure and haemolysis, and increased mortality ${ }^{3}$. The gold standard treatment for severe PVL has been treated surgically. However, two studies suggested that re-intervention can cause a high mortality rate ${ }^{45}$.

The long-term outcomes and functionality after transcatheter aortic-value replacement (TAVR) were investigated in 2032 patients (from 57 different centres) who had aortic stenosis. The patients were randomly assigned to either the TAVR group or the surgical replacement treatment group ${ }^{6}$. In this study, PVL was reported to be higher in TAVR cohort (TAVR: $33.3 \%$ and Surgical treatment: $6.3 \%$ ). Those in the TAVR group also had more hospitalizations and aortic valve re-interventions than those in the surgery group (TAVR: $33.3 \%$ and Surgical treatment: $25.2 \%$, hazard ratio of 1.28, 95\% confidence interval, 1.07 to 1.53 ). Despite the TAVR group having more undesirable outcomes, a similar health status improvement was seen in both groups ${ }^{6}$.

\section{Classification of $P V L$}

PVL can be graded using mainly three different grading systems: the 3-class, 4-class, and 5-class systems. The 3-class system involves grading PVL severity using the categories; mild, moderate, and severe ${ }^{7}$. Whereas the 4-class system involves grading PVL into grade 1, 2, 3, and 4. As some PVLs may fall into 'in-between' groups, it is difficult to place them into categories, for example a PVL may be not mild nor moderate so cannot be placed into a group. For this precise reason, it may be advisable to grade PVLs using the 5-class system that covers the 'in-between' groups; the categories are: mild, mild to moderate, moderate, moderate to severe and severe. Some argue that the presence of more classification categories will create inconsistency in the classification. However, others argue that this system brings unification and clarification to the classification system ${ }^{7}$.

The major structural parameters that govern the grading of the PVL include: the shape, size, number, location of the PVL defect, the anatomical landmarks present, and the size of the left atrium and ventricle. 2dimensional (2D) echocardiography can be used to assess the dehiscence and severity of PVL associated with the aortic value, whilst 3D echocardiography is usually used pre-operatively/intra-operatively for anatomical localization. PVLs that are graded 'moderate to severe' or 'severe' are usually due to a poorly placed valve or calcium build up in between the stent and the native annulus. The increase in left ventricle size can be a good indicator of chronic PVL (a PVL that have been present for more than three months), as those with acute PVL do not have a significant change in ventricle size or functionality. A mild, moderate, severely enlarged ventricle can indicate a mild, moderate, or severe PVL respectively ${ }^{7}$. Using qualitative echocardiography, it can be seen that in mild PVLs, the colour doppler jet width is less than $25 \%$ of the left ventricular width ${ }^{8}$. Whilst, in the moderate and severe PVL, the jet width is $25-60 \%$ and more than $60 \%$ less than the left ventricular width respectively. The severity can also be classified using semi-qualitative echocardiography- 
which uses the total of the PVL jet circumference divided by the valve circumference as the circumferential extent. Mild PVLs have a circumferential extent of less than 10\%, moderate PVLs extent is between 10-30\% and severe PVLs have an extent that is more than $30 \%^{8}$.

Diagnosis and evaluation of $P V L$

Investigation tools such as transthoracic echocardiography (TTE), transoesophageal echocardiography (TOE), cardiac computerised tomography (CT) and cardiovascular magnetic resonance (CMR) are used to diagnose PVLs. The tool used is selected based on the outcome required and the patient's health status. Following are the advantages and disadvantages of each imaging modality; a summary can also be found in Table 1 .

\section{Echocardiogram}

The first line diagnosis method for suspected PVL, alongside clinical assessment and physical examination, involves transthoracic echocardiogram (TTE). The non-invasive method provides evaluation on the functionality of the prosthetic valve. The prosthetic valve is assessed using $2 \mathrm{D}$ imaging techniques and doppler. This assessment is used to determine factors such as: the size of both the atria and ventricles, valvular disease and the possible masses that may be present in either the original or prosthetic valve. Although there are benefits of using TTE, its use is restricted by shadowing seen on images, which may be caused by the prosthetic valve components or calcification of the annulus. The inaccurate imaging can result in the doppler signal having no colour ultimately resulting in underestimation of the severity of PVL and poor aetiology differentiation ${ }^{9}$. An example of a patient's scan with aortic PVL can be seen in Figure $\mathbf{1}^{10}$.

Transesophageal echocardiogram (TEE) is an invasive imaging technique, preferred for intraprocedural guidance, that can be used to accurately determine the severity, mechanism, and the location of the PVL. In addition to this, TEE can be used to visualise any tears in the prosthetic valve. Regardless of the benefits, like TTE, this imaging modality can also be limited by shadowing due to anterior aortic annulus ${ }^{9}$. TEE's use is restricted due to it being an invasive procedure that requires trained physicians, patient sedation, mechanical ventilation for the patient and a possible injury to the oesophagus ${ }^{11}$.

\section{Cardiac $C T$}

In cases where both TTE and TEE cannot determine conclusively the grade or location of the PVL, cardiac CT can be used ${ }^{9}$. Cardiac CT is usually used as an imaging technique preoperatively for those who are due for TAVR; it can aid in verifying the annulus' size and shape and calcification degree ${ }^{12}$. Multiple studies which have investigated the effect of calcification on the presence of PVLs have found a correlation between calcification, which can only be removed by surgically, and aortic regurgitation post TAVI ${ }^{13} 14$. The downfalls of cardiac CT scanning, however, need to be considered before a patient undergoes it. Cardiac CT involves the use for intravenous iodinated contrast agents which can potentially cause nephropathy ${ }^{15}$ and anaphylactic shock (which is rare but serious life-threatening condition that requires urgent care) ${ }^{16}$. The patient will be exposed to radiation and poor resolution is seen in those with tachycardia ${ }^{9}$. There is also a need for a specialist radiologist report after a CT is performed which may be time consuming and that is not ideal in an emergency setting. In emergency cases, it would be advisable to use echocardiography instead as it can be easily read by cardiologists and surgeons ${ }^{17}$.

\section{Cardiac magnetic resonance (CMR)}

CMR can also be used in all patients especially when the TTE and TEE cannot conclusively predict the PVL degree or location in patients ${ }^{9}$. As mentioned previously, there is a risk of nephropathy when using $\mathrm{CT}$ in patients with renal failure. CMR is a novel non-contrast imaging modality that can be used in elderly patients ${ }^{18}$. This imaging technique can also be used in all patients, even those with mechanical valve prostheses if the imager has the necessary equipment. A major limitation seen with CMR is the overestimation of the severity of the PVL. To overcome this hurdle, it is necessary to utilise other imaging in assessing the severity of PVL. Due to the components of the mechanical valve, CMR also provides a poor image with mechanical valves in comparison to bioprosthetic valves. In addition to this, the assessment 
accuracy is also affected by tachyarrhythmias ${ }^{9}$ and presence of claustrophobia in some patients (usually due to the nature of the technique or the duration of the procedure) ${ }^{19}$ In those who are claustrophobic, a relaxant such as diazepam could be used to obtain a successful image ${ }^{19,20}$.

\section{Clinical impact of PVL:}

The presence of PVL may cause many major consequences including clinical and haemodynamic complications that reduces the long-term survival. Clinical presentation of PVL is primarily due to heart failure ${ }^{12}$. Chronic regurgitation can cause left atrial and ventricular pressure increase and volume overload including symptoms of pulmonary oedema and heart failure. Whilst a less severe regurgitation can only cause heart failure should the receiving chamber be non-compliant, making it difficult to attribute the patients' symptoms to the PVL. ${ }^{21}$ Patients with small PVLs are more likely to experiences symptoms of haemolytic anaemia (significant anaemia is usually treated with blood and/or platelet transfusion) whereas those with larger PVLs have symptoms of heart failure and infectious endocarditis. ${ }^{22}$ Recent studies have suggested that moderate to severe PVL occurs in $2.5 \%$ of patients after the TAVR ${ }^{23}$. It was believed that moderate to severe PVL is more likely to cause mortality. However, a Placement of AoRTic TraNscathetER valve (PARTNER) trial had proven that even a mild PVL is connected with increased mortality ${ }^{24}$, rejecting this theory.

\section{Available management options}

PVL can be treated through an invasive open-heart surgery or through a catheter-based treatment. As PVLs vary in shape and size, the correct device must be used to ensure successful closure of PVLs. There are 6 most frequent devices that are used to manage paravalvular leak. The devices, seen in Figure 2 , include Amplatzer vascular plugs (AVP2, AVP3 and AVP4), Amplatzer Duct Occluder (ADO), muscular Ventricular Septal Defect Occluder (mVSD) and Paravalvular Leak Device (PLD) ${ }^{25}$. The properties of the device vary by material, occlusive planes, waist length, size difference (between the waist and the disk) and are summarized in Table 2 . The device selected for use depends on the factors such as the distance of the landing zones, the size of the PVL, and the type of blood flow at the location of the PVL. AVP1, for example, is used in short landing zones and, since it can be repositioned, it also provides a precise and rapid occlusion whereas AVP2 can be utilised in variable landing zones. In situations where the PVL is in a high blood flow area or in demanding wall apposition cases, AVP3 can be used. In situations where the anatomy is tortuous, it is best to use AVP4. The use of AVP relies on the patient having an effective coagulation system. Should the patients have an ineffective coagulation system, AVP might not provide complete occlusion. The delivery of the AVP also requires a guiding catheter and large access sheath and as the delivery wires are hard, it may also be challenging to guide the device to the appropriate area ${ }^{26}$.

\subsection{Surgical management}

Open heart surgery is a gold standard treatment provided for significant and complex PVLs; it either aims to repair or replace the valve ${ }^{27}$. The surgical strategy is decided based on factors such as patient status, the aetiology, the condition of the native annulus, the size and location of the leak and whether the area is accessible ${ }^{27}$. The use of open surgery is reduced primarily due to the high morbidity and mortality rates $^{28}$. The rate of recurrence of paravalvular leaks is also common as the underlying pathology is not really treated. Open-heart surgery is, therefore, performed in cases where: the execution of percutaneous treatment is contraindicated (e.g. if the patient presents with active endocarditis), where the prosthetic valve is dysfunctional ${ }^{28}$, or in those who have severe PVLs (where performing an open-heart surgery has shown improved survival and reduced symptoms in patients) ${ }^{27}$.

\section{Transcatheter aortic valve closure}

The use of percutaneous catheter-based methods and other minimally invasive procedures have developed a great deal of curiosity and interest with regards to treating PVL and resultant regurgitation as not only do they treat the PVL but also reduce risk of further re-operation. To perform the percutaneous method, you need special occlusive devices and access to the site. The access to the site varies according to what 
type of PVL is present; mitral or aortic PVL. Aortic PVL is accessed through the femoral artery with a retrograde approach. Mitral PVL is accessed through the femoral vein and a transseptal puncture ${ }^{28}$. Whilst performing these procedures, it is important that the patient is administered general anaesthesia for their comfort and because there may be long-lasting equipment dwell ${ }^{27}$. PVL closures can be performed using three approaches (transseptal, retrograde transaortic or left ventricle transapical approaches) and the approach taken is decided based on the location of leak, the valve involved, presence of any hinderance or calcification. Aortic paravalvular leaks are treated using the retrograde transaortic approach.

A hydrophilic guidewire, supported by a catheter, is initially directed towards the PVL and either echocardiography or fluoroscopy is used to confirm paravalvular position. The catheter is then inserted into the left ventricle through the defect. Whilst taking extra precautions, the hydrophilic wire is subsequently replaced with an extra support wire; the precautions are necessary to prevent cardiac perforation risk. A delivery sheath for the device is introduced into the left ventricle and then pulled into the defect. The correct placement of the device is confirmed by using $\mathrm{TOE}^{27}$. An overview of a protocol can be seen pictorially in Figure 3 .

Despite the retrograde transaortic approach being the most ideal for an aortic PVL, in some cases the transapical approach may be used. The transapical approach may be necessary when a mitral or aortic mechanical prosthesis is present or when the retrograde transaortic approach is not successful. The antegrade transseptal approach requires an exteriorized arteriovenous (AV) for support and control of the device and sheath during delivery. When the crossing wire is inserted into the descending aorta, snared, and then exteriorized through the femoral arterial sheath, the AV rail is created. It's important to avoid putting too much pressure on the left ventricle loop, which can entrap the anterior mitral leaflet and produce severe mitral regurgitation ${ }^{27}$.

\section{Outcomes of PVL management}

Surgical treatment of mild and moderate PVLs with no adverse effects is successful in almost $80-90 \%$ of patients ${ }^{29}$. Complications due to treatment using the transcatheter method is rare. Studies that investigated the emergence of adverse effects in 115 patients noticed that in a 30-day period post treatment, $1.7 \%$ had an unexplained or sudden death, $2.6 \%$ had a stroke, $0.9 \%$ required an emergency surgery and $5.2 \%$ required treatment for periprocedural bleeding ${ }^{30} 31$. Two studies had also noticed that $2.5 \%$ of patients had experienced device embolism and death was seen in approximately $0.5 \%$ of patients ${ }^{3031}$.

The transcatheter method may not be successful in some patients. This can be due to impingement of the prosthetic leaflet and the inability to guide the catheter or cross the defects of the wire. Although it is more common for impingement to occur in patients with mechanical valves, it can occur with any prosthesis. Impingement can be prevented with the use of smaller devices but can still be challenging with the nature of the shape of occluders and the location, with regards to the surgical annular ring. Clinical success and the symptoms experienced by patients is dependent upon the degree of regurgitation; it is also seen that those who have heart failure suffer a greater deal of symptoms compared to those with haemolysis ${ }^{32}$.

In a study looked at the risk of death and developing heart failure with rehospitalization in 1661 patients after 1-year post-TAVR. A 9.3\% of the patients had died within the year whilst approximately $15 \%$ of the patients had hospitalized with heart failure. Those with the least moderate form of PVL also had the highest mortality rate. It was also seen that the degree of PVL had decreased by one class/group in almost $80 \%$ of patients ${ }^{33}$. Another study evaluated the outcomes seen after 3 years and saw that there was a survival rate of $64 \%$, cardiac-associated death rate of $9.5 \%$, non-cardiac associated death rate of approximately $10 \%$. $72 \%$ of those who survived were symptom free and did not require any other surgery ${ }^{32}$.

\section{Conclusion}

Patients with paravalvular leak should be assessed through multi-disciplinary team approach and patientselective plan should be in place. Open surgical intervention is reserved for complex cases where minimal invasive techniques cannot be utilized. 
Ethical approval: no ethical approval or IRB required

Trial registration: not applicable for this study

References

1. Goel K, Eleid MF. Paravalvular Leak in Structural Heart Disease.Curr Cardiol Rep . 2018;20(3). doi:10.1007/s11886-018-0959-x

2. Wojakowski W, Smolka G. Paravalvular leak - important complication after implantation of prosthetic valve. E-Journal ESC Counc Cardiol Pract . 2010;9(8):1-7.

3. García E, Arzamendi D, Jimenez-Quevedo P, et al. Outcomes and predictors of success and complications for paravalvular leak closure: An analysis of the Spanish realwOrld paravalvular LEaks closure (HOLE) registry. EuroIntervention . 2017;12(16):1962-1968. doi:10.4244/EIJ-D-16-00581

4. Dávila-Román VG, Waggoner AD, Kennard ED, et al. Prevalence and severity of paravalvular regurgitation in the Artificial Valve Endocarditis Reduction Trial (AVERT) echocardiography study. J Am Coll Cardiol . 2004;44(7):1467-1472. doi:10.1016/j.jacc.2003.12.060

5. Echevarria JR, Bernal JM, Rabasa JM, Morales D, Revilla Y, Revuelta JM. Reoperation for bioprosthetic valve dysfunction. 2018;(July):523-527.

6. Raj R. Makkar, M.D., Vinod H. Thourani, M.D, et al. Five-Year Outcomes of Transcatheter or Surgical Aortic-Valve Replacement. Published online 2020. doi:10.1056/NEJMoa1910555

7. Pibarot P, Hahn RT, Weissman NJ, Monaghan MJ. Assessment of paravalvular regurgitation following TAVR: A proposal of unifying grading scheme. JACC Cardiovasc Imaging . 2015;8(3):340-360. doi:10.1016/j.jcmg.2015.01.008

8. Hartlage GR, Babaliaros VC, Thourani VH, et al. The role of cardiovascular magnetic resonance in stratifying paravalvular leak severity after transcatheter aortic valve replacement: an observational outcome study. J Cardiovasc Magn Reson . 2014;16(December):93. doi:10.1186/s12968-014-0093-x

9. Fanous EJ, Mukku RB, Dave P, et al. Paravalvular Leak Assessment: Challenges in Assessing Severity and Interventional Approaches.Curr Cardiol Rep . 2020;22(12). doi:10.1007/s11886-020-01418-7

10. Gafoor S, Franke J, Bertog S, et al. A quick guide to paravalvular leak closure. Interv Cardiol Rev . 2015;10(2):112-117. doi:10.15420/icr.2015.10.2.112

11. J. Heiberg, D. El-Ansary, C. F. Royse, A. G. Royse, A. A. Alsaddique DJC. Transthoracic and transoesophageal echocardiography: a systematic review of feasibility and impact on diagnosis, management and outcome after cardiac surgery. Published online 2016. doi:https://doi.org/10.1111/anae.13545

12. Binder RK, Webb JG, Willson AB, et al. The impact of integration of a multidetector computed tomography annulus area sizing algorithm on outcomes of transcatheter aortic valve replacement: A prospective, multicenter, controlled trial. J Am Coll Cardiol . 2013;62(5):431-438. doi:10.1016/j.jacc.2013.04.036

13. Ewe SH, Ng ACT, Schuijf JD, et al. Location and severity of aortic valve calcium and implications for aortic regurgitation after transcatheter aortic valve implantation. Am J Cardiol . 2011;108(10):1470-1477. doi:10.1016/j.amjcard.2011.07.007

14. Daniel John 1, Lutz Buellesfeld, Seyrani Yuecel, et al. Correlation of Device landing zone calcification and acute procedural success in patients undergoing transcatheter aortic valve implantations with the selfexpanding CoreValve prosthesis. doi:10.1016/j.jcin.2009.11.015

15. Suchá D, Symersky P, Tanis W, et al. Multimodality Imaging Assessment of Prosthetic Heart Valves. Circ Cardiovasc Imaging . 2015;8(9):1-14. doi:10.1161/CIRCIMAGING.115.003703 
16. Halna du Fretay X, Gaillet G, Hoarau C, Blanchard-Lemoine B. Choc anaphylactique à un produit de contraste iodé: Intérêt du bilan allergologique à propos d'un cas. Ann Cardiol Angeiol (Paris) . 2012;61(6):457461. doi:10.1016/j.ancard.2012.09.007

17. Sun Z. Cardiac CT imaging in coronary artery disease: Current status and future directions. Quant Imaging Med Surg . 2012;2(2):98-105. doi:10.3978/j.issn.2223-4292.2012.05.02

18. Rogers T, Waksman R. Role of CMR in TAVR. JACC Cardiovasc Imaging . 2016;9(5):593-602. doi:10.1016/j.jcmg.2016.01.011

19. Francis JM, Pennell DJ. Treatment of claustrophobia for cardiovascular magnetic resonance: Use and effectiveness of mild sedation. J Cardiovasc Magn Reson . 2000;2(2):139-141. doi:10.3109/10976640009148683

20. Bernard S, Yucel E. Paravalvular Leaks-From Diagnosis to Management. Curr Treat Options Cardiovasc Med . 2019;21(11). doi:10.1007/s11936-019-0776-6

21. Eleid MF, Cabalka AK, Malouf JF, Sanon S, Hagler DJ, Rihal CS. Techniques and outcomes for the treatment of paravalvular leak.Circ Cardiovasc Interv . 2015;8(8):1-13. doi:10.1161/CIRCINTERVENTIONS.115.001945

22. Ruiz CE, Hahn RT, Berrebi A, et al. Clinical Trial Principles and Endpoint Definitions for Paravalvular Leaks in Surgical Prosthesis: An Expert Statement. J Am Coll Cardiol . 2017;69(16):2067-2087. doi:10.1016/j.jacc.2017.02.038

23. Vemulapalli S, Carroll JD, Mack MJ, et al. Procedural Volume and Outcomes for Transcatheter AorticValve Replacement. N Engl J Med . 2019;380(26):2541-2550. doi:10.1056/nejmsa1901109

24. Kodali SK, Williams MR, Smith CR, et al. Two-Year Outcomes After Transcatheter or Surgical Aortic Valve Replacement. Surv Anesthesiol . 2013;57(4):166-167. doi:10.1097/01.sa.0000431219.37059.d1

25. Calvert PA, Northridge DB, Malik IS, et al. Percutaneous device closure of paravalvular leak. Circulation . 2016;134(13):934-944. doi:10.1161/CIRCULATIONAHA.116.022684

26. Zander T, Medina S, Montes G, Nuñez-Atahualpa L, Valdes M, Maynar M. Endoluminal occlusion devices: Technology update. Med Devices Evid Res . 2014;7:425-436. doi:10.2147/MDER.S49540

27. Kliger C, Eiros R, Isasti G, et al. Review of surgical prosthetic paravalvular leaks: Diagnosis and catheterbased closure. Eur Heart J . 2013;34(9):638-648. doi:10.1093/eurheartj/ehs347

28. Branny M, Januška J, Škňouřil L, Holek B, Dorda M, Gajdůšek L. Management of paravalvular leaks. Cor Vasa . 2012;54(5-6):235-238. doi:10.1016/j.crvasa.2012.05.013

29. Ruiz CE, Jelnin V, Kronzon I, et al. Clinical outcomes in patients undergoing percutaneous closure of periprosthetic paravalvular leaks.J Am Coll Cardiol . 2011;58(21):2210-2217. doi:10.1016/j.jacc.2011.03.074

30. Lerakis S, Hayek S, Arepalli CD, et al. Cardiac magnetic resonance for paravalvular leaks in post-transcatheter aortic valve replacement.Circulation . 2014;129(14):430-431. doi:10.1161/CIRCULATIONAHA.113.003683

31. Sorajja P, Cabalka AK, Hagler DJ, Rihal CS. Percutaneous repair of paravalvular prosthetic regurgitation: Acute and 30-day outcomes in 115 patients. Circ Cardiovasc Interv . 2011;4(4):314-321. doi:10.1161/CIRCINTERVENTIONS.110.960955

32. Sorajja P, Cabalka AK, Hagler DJ, Rihal CS. Long-term follow-up of percutaneous repair of paravalvular prosthetic regurgitation. J Am Coll Cardiol . 2011;58(21):2218-2224. doi:10.1016/j.jacc.2011.07.041

33. Pibarot P, Hahn RT, Weissman NJ, et al. Association of paravalvular regurgitation with 1-year outcomes after transcatheter aortic valve replacement with the SAPIEN 3 valve. JAMA Cardiol . 2017;2(11):12081216. doi:10.1001/jamacardio.2017.3425 
34. Park J, Suradi HS. State-of-the-art Structural Interventions in Heart Failure. Card Fail Rev . 2019;5(3):147-154. doi:10.15420/cfr.2019.12.2

\section{Figures}

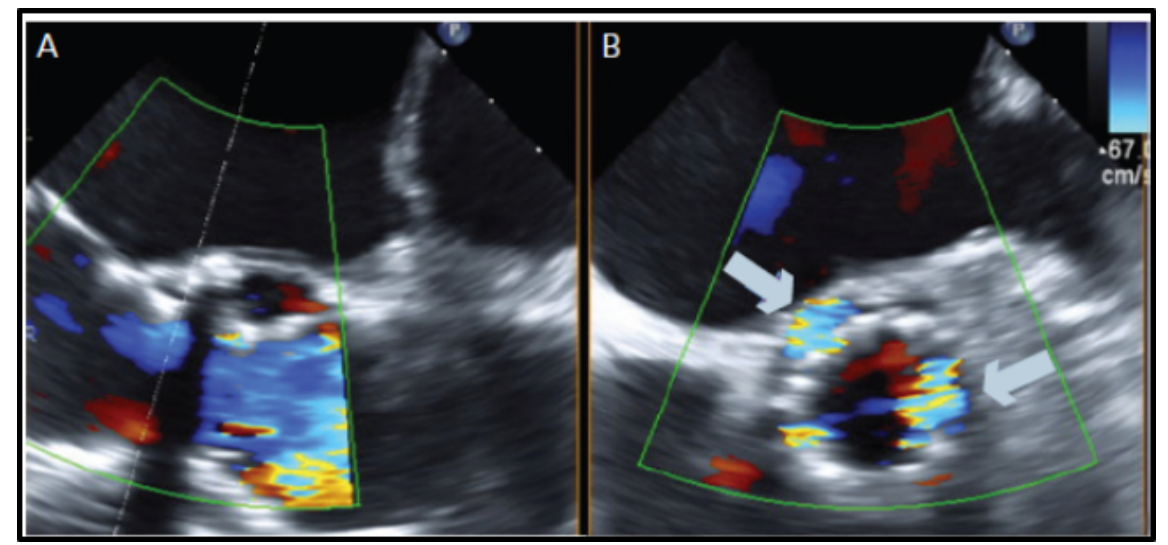

Figure 1 shows adapted TTE images from Gafoor et al of a patient with an aortic PVL. (A) is a 120 degree view of the aortic valve whilst (B) shows a biplane view. (B) shows two areas of major leak (indicated by the blue arrows $)^{10}$.

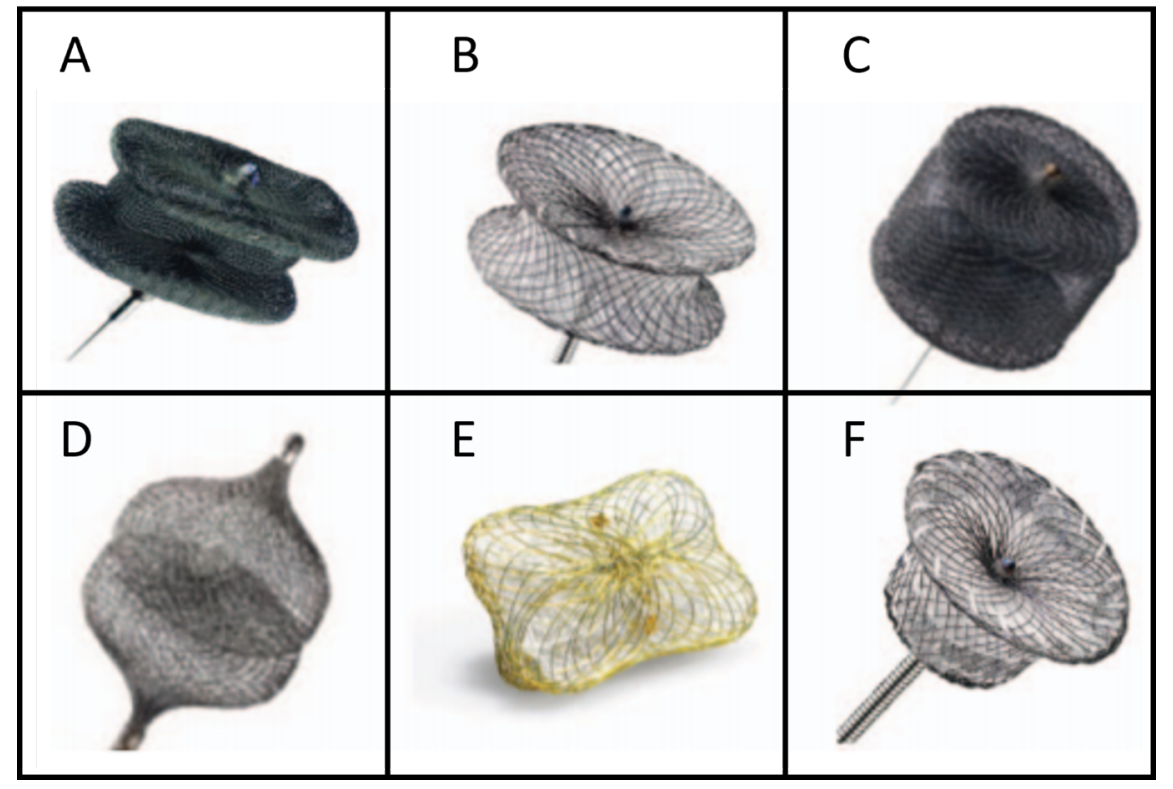

Figure 2 shows the different types of devices that can be used to manage PVL. A, B, C, D, E and F are AVP3, mVSD, AVP2, AVP4, PLD and ADO respectively ${ }^{25}$. 


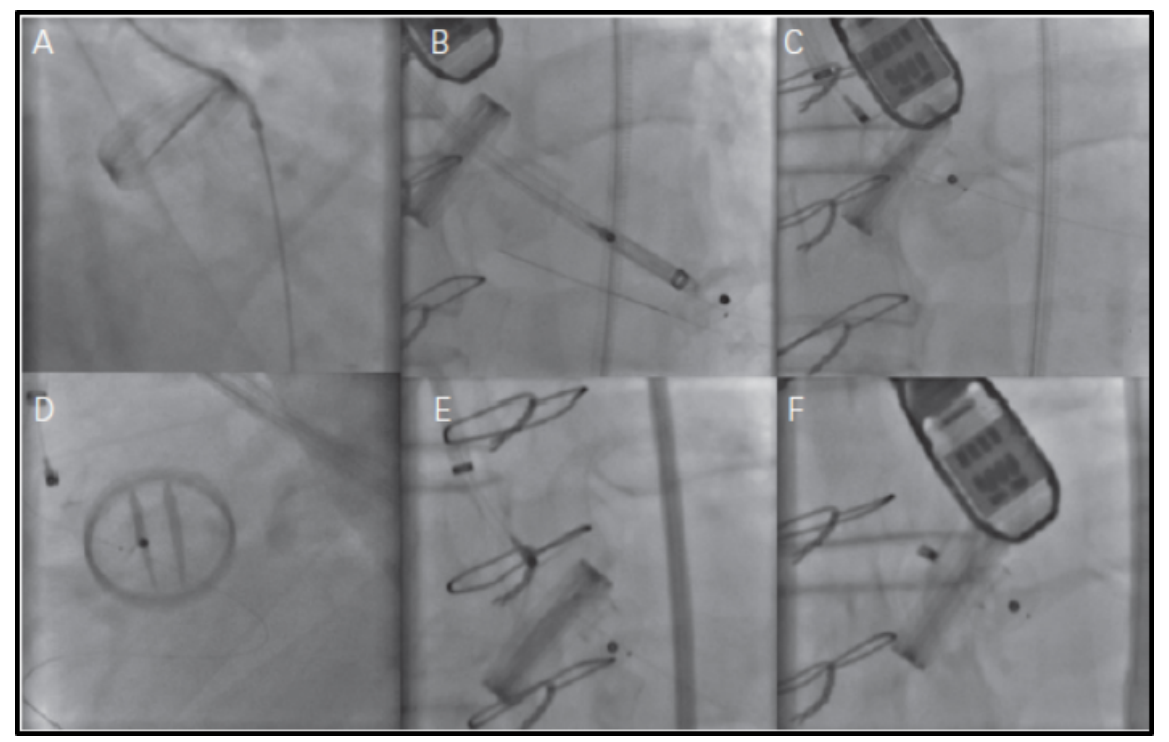

Figure 3 shows x-rays with contrast demonstrating the transcatheter aortic valve closure procedure. (A) shows the hydrophilic wire, a retrograde fashion, being used to cross the PVL. (B) shows the wire being replaced by a shuttle sheath and then placed into the left ventricle. (C) demonstrates the failed attempt of closing the leak with an occluder device. (D) shows the wire in place. (E) shows an AVP3 being placed across the leak. (F) shows the device being released after confirmation of minimal leak and optimum functionality using echocardiography ${ }^{34}$.

\section{Tables}

Table 1 Advantages and disadvantages with the use of different imaging modalities.

\begin{tabular}{ll}
\hline Technique & Advantages \\
TTE & Non-invasive method that provides evaluation on prosthetic valve function. \\
& Can provide volumetric quantitation. \\
& It is cheaper as it requires less costly resources. \\
TOE & Accurately determines the severity, mechanism, and location of the PVL. \\
& Best imaging method to visualise prosthetic valve tears.
\end{tabular}

Cardiac CT Can be used preoperatively to identify the size and shape of the annulus and calcification degree to reduce

Cardiac MRI It can be used in patients with both mechanical and prosthetic valves.

The images obtained have good quality.

There is non-need for the use contrasting agents.

Table 2 Different devices that can be used to manage paravalvular leak

\begin{tabular}{lllllll}
\hline & Types of & Types of & Types of & Types of & Types of \\
& Devices & Devices & Devices & Devices & Devices \\
Variables & AVP2 & AVP3 & AVP4 & ADO & mVSD \\
& $\begin{array}{llll}\text { Polyester cloth } \\
\text { Occlusive }\end{array}$ & No & No & No & Yes & Yes \\
& planes & & 4 & 4 & 3 & 4
\end{tabular}




$\begin{array}{llllll}\text { Waist length } & 6 \mathrm{~mm} & 2-5 \mathrm{~mm} & 4-8 \mathrm{~mm} & \begin{array}{l}5-8 \mathrm{~mm} \\ 4 \mathrm{~mm} / 6 \mathrm{~mm}\end{array} & 7 \mathrm{~mm} \\ \begin{array}{l}\text { Size } \\ \text { difference }\end{array} & \text { No } & 2 \mathrm{~mm} & 2 \mathrm{~mm} & \begin{array}{l}\text { (depending } \\ \text { on the type } \\ \text { of APO) }\end{array}\end{array}$

\section{Hosted file}

Aortic PVL figures.tiff available at https://authorea.com/users/435050/articles/538342-themanagement-of-paravalvular-leaks-post-aortic-valve-replacement

\section{Hosted file}

Aortic PVL Tables.docx available at https://authorea.com/users/435050/articles/538342-themanagement-of-paravalvular-leaks-post-aortic-valve-replacement 